\title{
Angewandte Kritische Geographie am Beispiel der Wohnungsmarktforschung in Göttingen
}

\author{
Michael Mießner ${ }^{1}$ iD \\ Eingegangen: 6. April 2020 / Überarbeitet: 20. Juli 2020 / Angenommen: 13. August 2020 / Online publiziert: 22. September 2020 \\ (c) Der/die Autor(en) 2020
}

\section{Zusammenfassung}

Der Beitrag widmet sich der anwendungsbezogenen Forschung, die erst jüngst in den Fokus der Kritischen Geographie rückte. Der Aufsatz greift diese Forschungslücke auf und erläutert die grundsätzliche Ausrichtung einer angewandten Kritischen Geographie. Dies beinhaltet die Entwicklung von theoretischen Konzepten zum Verständnis sozialer Entwicklungen, die Untersuchung von Problemlagen marginalisierter Bevölkerungsgruppen und die Erforschung von Fragstellungen, die über den universitären Kontext hinaus insbesondere für soziale Bewegungen relevant sind. Darauf aufbauend stellt der Beitrag meine eigene Forschung zum Göttinger Wohnungsmarkt vor. Die Entwicklungen auf dem Wohnungsmarkt können theoretisch als finanzgetriebene kaskadenförmige Ausbreitung der Immobilieninvestitionen sowie als eine Form der Studentifizierung gefasst werden. Die Untersuchungen über Mietpreisentwicklungen und Segregationsprozesse in der Stadt ermöglichte ein breites Verständnis von wichtigen Entwicklungen, die insbesondere einkommensschwache Bevölkerungsgruppen betreffen. Der Wissenstransfer der Forschungsergebnisse in lokalen Medien führte dazu, dass die ablaufenden Prozesse in der Bevölkerung bemerkt und stadtpolitisch, sowohl seitens der Stadtverwaltung und des Stadtrates als auch von zivilgesellschaftlichen Initiativen adressiert wurden. Zugleich konnten in der Zusammenarbeit mit Akteuren aus sozialen Bewegungen diesen Akteuren wichtige Informationen für ihr politisches Handeln zur Verfügung gestellt werden. Die vorgestellte Arbeit macht auch 3 Herausforderungen angewandter kritischer Forschung deutlich. Die Zusammenarbeit mit Akteuren sozialer Bewegungen ist zeitaufwendig. Zudem besteht häufig eine Diskrepanz zwischen den Logiken der Generierung wissenschaftlichen Wissens und den zu untersuchenden lokalen Logiken sozialer Entwicklungen. Dennoch muss sich eine angewandte Kritische Geographie der Herausforderung stellen, auf politische Entscheidungen zugunsten der Verbesserung der Lebensbedingungen marginalisierter Bevölkerungsgruppen Einfluss zu nehmen.

Schlüsselwörter Kritische Wohnungsmarktforschung $\cdot$ Gentrifizierung $\cdot$ Studentifizierung $\cdot$ Segregation $\cdot$ Soziale Wohnungspolitik

Michael Mießner

michael.miessner@tu-dresden.de

1 Institut für Geographie, Technische Universität Dresden, Helmholtzstr. 10, 01069 Dresden, Deutschland 


\begin{abstract}
This article is devoted to applied research, which came into focus in critical geography only recently. The article takes this research gap and explains the basic characteristics of applied critical geography. This includes developing theoretical concepts for understanding important social developments, investigating problems of marginalised social groups, as well as investigating research questions that go beyond academic contexts and are especially relevant to social movements. On this basis, this article explains my own research on the Goettingen housing market. The developments in the Goettingen housing markets can be theoretically expressed as finance-driven, cascade-like expansion of real-estate investment, as well as a form of studentification. The investigation of the rent price developments and the processes of urban segregation allows an understanding of important developments that particularly affect low-income households. The knowledge-transfer of research outcomes in local media led to the processes taking place being noticed by the population and addressed in urban policy by the city administration and the city council, as well as by civil society initiatives. Furthermore, in cooperation with stakeholders from social movements important information could be made available to them for their political actions. The article presented distinguishes three challenges of applied critical research. Cooperation with stakeholders from social movements is very time-consuming. Additionally, there is a discrepancy between the logic of scientific knowledge creation and the local logic of social development to be investigated. However, applied critical geography has to meet the challenge of influencing political decisions for the benefit of improving the living conditions of marginalised groups of the population.
\end{abstract}

Keywords Critical housing studies $\cdot$ Gentrification $\cdot$ Studentification $\cdot$ Segregation $\cdot$ Social housing policy

\section{Einleitung}

Anwendungsbezogene Forschung spielte lange Zeit eine untergeordnete Rolle in der Kritischen Geographie. Ausgehend von der marxschen Analyse der politischen Ökonomie entstanden Untersuchungen über Ursachen und Bedingungen der kapitalistischen Stadtentwicklung. Viele Forschungen waren, trotz ihres emanzipatorischen Anspruches, durch eine recht große Distanz zu sozialen Bewegungen gekennzeichnet (Kemper und Wiegand 2018). In jüngster Vergangenheit änderte sich dies zum Teil und es gibt erste Diskussionen, was eine angewandte Kritische Stadtgeographie ausmacht und in welchem Verhältnis sie zu sozialen Bewegungen stehen könnte (Schipper 2017). Zugleich spitzt sich die Wohnungsfrage in den letzten Jahren in vielen deutschen Städten erneut zu. Politisch bearbeitet wird sie dabei aber nur sehr selten explizit zur Verbesserung der Lebensbedingungen marginalisierter Bevölkerungsgruppen. Letzteres ist ein wichtiges Anliegen einer angewandten Kritischen Geographie (ibid.). Entsprechend ist es auch gesellschaftspolitisch für eine Kritische Geographie an der Zeit, anwendungsbezogene Aspekte verstärkt in ihre Forschungen einfließen zu lassen.

In einem solchen Feld bewegte sich auch meine eigene Forschung zu den Entwicklungen auf dem Göttinger Wohnungsmarkt und damit einhergehender Segregation und Verdrängung: Auf der einen Seite stand die theoriegeleitete Analyse der stattfindenden Prozesse. Auf der anderen Seite war ich von Beginn an bestrebt, die Forschungsergebnisse einer breiteren Öffentlichkeit zugänglich zu machen, zivilgesellschaftlichen Akteuren Interpretationsangebote für die Einordnung aktueller Entwicklungen bereitzu- stellen und auf stadtpolitische Entscheidungen zugunsten einer sozialeren Wohnungspolitik hinzuwirken.

Ich möchte das Sonderheft „Kritische Angewandte Geographie“ zum Anlass nehmen, meine Arbeiten zum Göttinger Wohnungsmarkt und zu den sozialräumlichen Prozessen in der Stadt aus der Perspektive einer angewandten Kritischen Geographie zu reflektieren. Dazu umreiße ich im Folgenden kurz, was eine angewandte kritische Forschung ausmacht. Im Anschluss daran stelle ich meine eigene Forschung über die Wohnungsmarkt- und stadtpolitische Entwicklung in Göttingen dar und werde im Fazit auf Möglichkeiten und Grenzen einer so ausgerichteten kritischen Wohnungsmarktforschung eingehen.

\section{Angewandte Kritische Geographie}

Kritisch-geographische Forschung hat in den vergangenen Jahren in der deutschsprachigen Forschung, insbesondere in der Stadtgeographie, erheblich an Aufmerksamkeit gewonnen (z.B. Belina et al. 2018; Oßenbrügge und Vogelpohl 2018). Schipper (2017, S. 5) kritisiert jedoch, dass der Fokus der Kritischen Geographie hauptsächlich auf der „Kritik des Bestehenden und der darin eingeschriebenen Machtverhältnisse“ liegt, sie aber wenig „Anknüpfungspunkte für emanzipatorische Transformationsprojekte" bietet.

In der Literatur werden 3 zentrale Aspekte einer angewandten Kritischen Geographie diskutiert (vgl. Jonas et al. 2015; Schipper 2017). Erstens gehe es darum, theoretische Konzepte zum Verständnis sozialer Prozesse und Beziehungen sowie deren Veränderung zu entwickeln, wie dies mit dem Begriff „Recht auf Stadt“ (Lefebvre 2016) gelungen 
ist, der auch von sozialen Bewegungen aufgegriffen wird. Zweitens müsse die Forschung sozial relevant und politisch engagiert sein und daher Entwicklungen untersuchen, die für marginalisierte Bevölkerungsgruppen von Relevanz sind. Dies ist z. B. in der Gentrifizierungsforschung üblich, die die Verdrängung von einkommensniedrigen Bevölkerungsgruppen herausarbeitet (z. B. Holm und Schulz 2016). Drittens muss die Forschung über den universitären Kontext hinaus relevant werden sowie Fragestellungen untersuchen, die für Akteure sozialer Bewegungen bedeutsam sind, von diesen selbst jedoch kaum mit eigenen Ressourcen beantwortet werden können.

\section{Angewandte kritische Wohnungsforschung in Göttingen}

Im Folgenden diskutiere ich meine Arbeiten zum Göttinger Wohnungsmarkt hinsichtlich der o.g. 3 Dimensionen einer angewandten Kritischen Geographie. In der Stadt Göttingen wohnen über 135.000 Einwohner*innen, deren Zahl seit 2010 um rund $5 \%$ gestiegen ist (GÖSIS 2019). An der Georg-August-Universität und den Göttinger Hochschulen sind ca. 34.000 Studierende immatrikuliert, von denen rund 22.000 in der Stadt wohnen (ibid.). Entsprechend spielt das Segment des studentischen Wohnens eine bedeutende Rolle auf dem Mietwohnungsmarkt (Mießner 2019). Zwischen 2013 und 2019 hatte die Stadt eine Preissteigerung der durchschnittlichen Angebotsmieten um mehr als $28 \%$, von $8,00 € / \mathrm{m}^{2}$ auf mittlerweile $10,26 € / \mathrm{m}^{2} \mathrm{zu}$ verzeichnen (Marlow und Mießner 2019).

\section{Theoretisierung der Göttinger Entwicklungen}

Über lange Zeit galten steigende Mieten und Investitionsdruck auf dem Immobilienmarkt als ein Phänomen, dass überwiegend deutsche Metropolen betraf (Heeg 2011). Kleinere Großstädte standen hingegen kaum im Forschungsfokus. Die steigenden Miet- und Immobilienpreise in einer Stadt wie Göttingen lassen sich dabei theoretisch konzeptionell mit Bezug auf 2 Debattenstränge, die Finanzialisierung von Wohnraum (Aalbers 2016) und die Studentifizierung (Smith 2005), fassen.

Erstens kommt es unter den Bedingungen des finanzdominierten Akkumulationsregimes (Sablowski 2011) zu vermehrten Investitionen in die gebaute Umwelt (Harvey 2006). Dabei konzentrieren sich die Investitionen zunächst auf die wachsenden Metropolen, weil diese als sicher gelten und hohe Renditen versprechen (Heeg 2011). Aufgrund des so induzierten Anlagedrucks beginnt die rent gap (Smith 1979), die Lücke zwischen der kapitalisierten und potenziellen Grundrente, in den Metropolen $\mathrm{zu}$ sinken. In der Folge setzt eine kaskadenförmige Ausbreitung (Lees 2006) der Immobilieninvestitionen ein und kleinere Großstädte, wie Göttingen, geraten in den Fokus von Immobilieninvestitionen (Fehlberg und Mießner 2015). Aus diesem Grund investieren seit einiger Zeit vermehrt finanzialisierte Immobilienunternehmen, wie z.B. Deutschlands sechstgrößtes Wohnungsunternehmen, die Adler Real Estate AG (Frieling et al. 2020), auf dem Göttinger Markt. Angesichts dieses Investitionsdruckes, steigen die Boden- und Immobilienpreise (Mießner und Klinge 2017). Gleichzeitig führt das Bevölkerungswachstum und die geringe Neubauquote $\mathrm{zu}$ einem angespannten Wohnungsmarkt (Frieling et al. 2020). Dies erhöht die Konkurrenz der Mieter*innen um Wohnraum, weshalb Vermieter*innen Mietpreissteigerungen leicht durchsetzen können (Mießner 2020b).

Zweitens können die in dieser Situation einsetzenden sozialräumlichen Verdrängungsprozesse als Studentifzierung (Smith 2005) bezeichnet werden. Das heißt nicht, dass Studierende aktiv andere Bevölkerungsgruppen verdrängen. Studentifizierung bedeutet vielmehr, dass Eigentümer*innen bevorzugt Wohnungen an Studierende vermieten, weil diese aus verschiedenen Gründen besonders als Mieter*innen attraktiv sind (Mießner 2019, 2020b). So sind z.B. durch das gemeinschaftliche Aufbringen der Miete in Wohngemeinschaften hohe Mietzahlungen möglich. Für studentische 1-Zimmer-Apartments können aufgrund der geringen Größe insgesamt erschwingliche Gesamtmieten gefordert werden, die Quadratmetermietpreise sind jedoch besonders hoch. Die hohe Fluktuation unter den Studierenden erleichtert regelmäßige Mieterhöhungen. Aus diesen Gründen bevorzugen viele Vermieter*innen Studierende gegenüber Familien und insbesondere Transferleistungsempfänger*innen sowie Menschen mit Migrationshintergrund. Auf solche Weise wird eine sozialräumliche Entmischung in Gang gesetzt, die als indirekte Verdrängung bezeichnet werden kann.

\section{Untersuchung der Mietpreisentwicklungen und Segregationsprozesse}

Angewandte Kritische Geographie untersucht Entwicklungen, die marginalisierte Bevölkerungsgruppen betreffen, wie z.B. steigende Mieten oder sozialräumliche Verdrängungsprozesse. Da keine verlässlichen Daten zur Mietpreisentwicklung in der Stadt vorlagen, habe ich die Entwicklung der Angebotsmietpreise für die Jahre 2013 bis 2019 erhoben und ausgewertet. So konnte ich erstens zeigen, dass der Anteil des preisgünstigen Mietsegmentes von unter $7 € / \mathrm{m}^{2}$ von knapp $30 \%$ auf mittlerweile weniger als $5 \%$ geschrumpft ist (vgl. Abb. 1). Damit waren 2019 wesentlich weniger Mietwohnungen für Geringverdiener*innen erschwinglich.

Zweitens konnte ich nachweisen, dass Stadtteile wie Grone oder die Weststadt besonders starke Steigerungsra- 


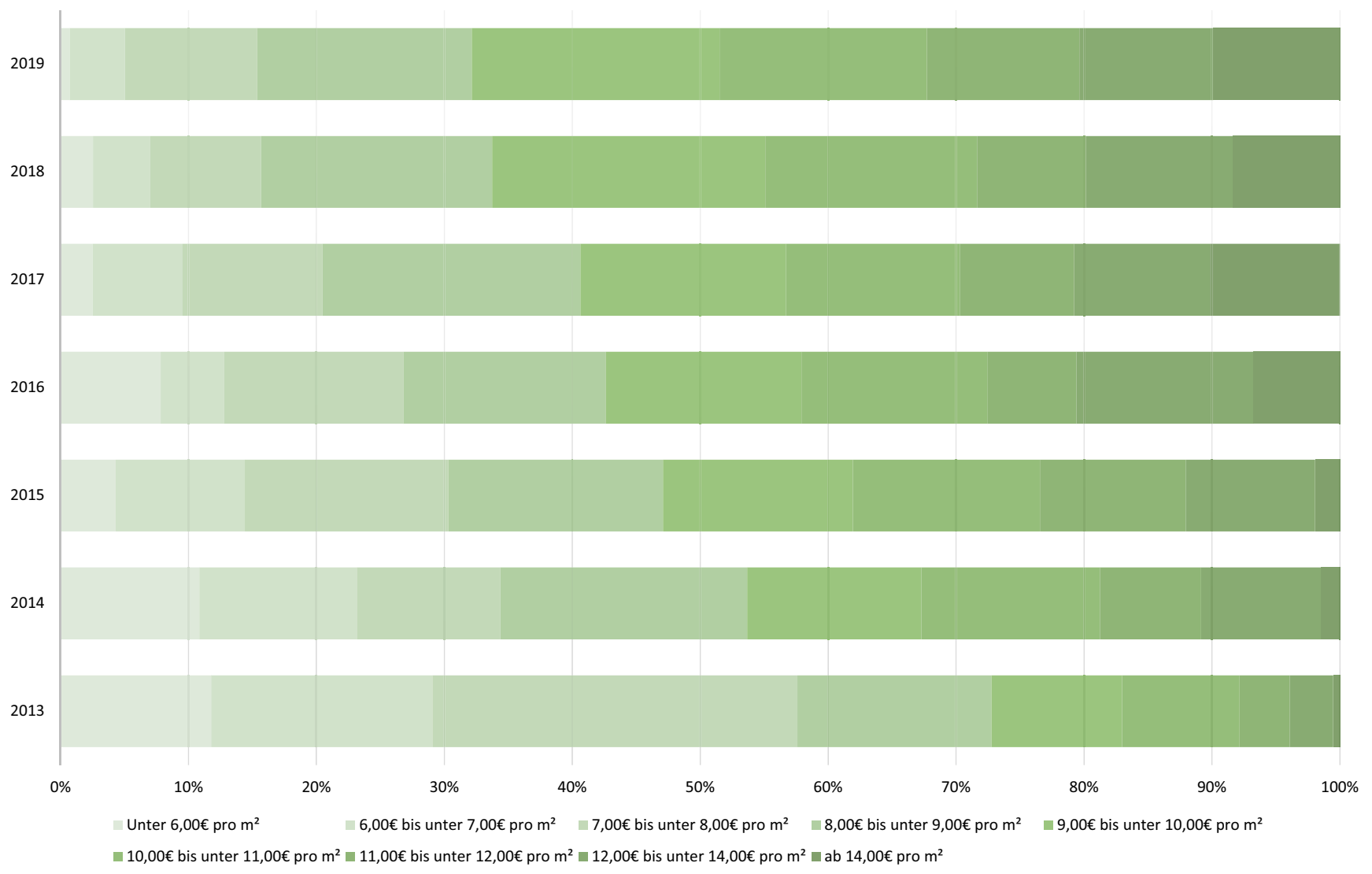

Abb. 1 Anteil der Wohnungsangebote in Göttingen nach Mietpreissegmenten 2013 bis 2018 (Zwei- und Mehrzimmerwohnungen). Quelle: Eigene Erhebungen, R. Marlow

ten aufweisen (vgl. Abb. 2 und 3). Diese Stadtteile waren anfangs durch geringe Mietpreise gekennzeichnet, zudem wohnen hier viele einkommensschwache Bevölkerungsgruppen (Mießner und Klinge 2017). Hohe Preissteigerungen weisen außerdem die Nordstadt und Weende auf, die sich durch einen hohen Anteil an Studierenden und ursprünglich hohe Mieten auszeichnen.

In Gesprächen mit Mieter*inneninitiativen entstand drittens die Idee, nachzuzeichnen, wie sich die Mieten in den Beständen einzelner größerer Vermieter*innen entwickeln. So konnte ich zeigen, dass in einzelnen Stadtteilen große Anbieter*innen wie Vonovia (Abb. 4) als erste ihre Mietpreise erhöhten und mit zeitlicher Verzögerung kleinere private Vermieter*innen die Mieten an diese Vonovia-Mieten anpassten. Damit kommt Großvermieter*innen eine entscheidende Rolle für die Mietpreisgestaltung zu (Mießner 2018).

Viertens nimmt die Segregation von Sozialleistungsempfänger*innen im Stadtgebiet zu. Sie konzentrieren sich in immer weniger Stadtteilen, v.a. der Weststadt und Grone (vgl. Abb. 5), die aktuell durch hohe Mietpreissteigerungen gekennzeichnet sind (Abb. 3). Das legt nahe, dass einkommensschwache Haushalte mit einer immer größeren Mietbelastung konfrontiert sind.

\section{Wissenstransfer}

In meinen Forschungen war es mir wichtig, Wissen zu generieren, dass auch für die lokale Bevölkerung relevant ist. Deshalb habe ich versucht, die Forschungsergebnisse mithilfe von Pressemeldungen und klassischen universitären Transfervorträgen einer breiten Öffentlichkeit zugänglich zu machen. Die Resonanz war sehr positiv. Es berichteten lokale Tageszeitungen, das Lokalradio und andere Medien (z.B. Göttinger Stadtinfo, Zeitschriften der Studierendenschaft). Darüber hinaus wurden die Ergebnisse von vielen im Stadtrat vertretenen Parteien aufgegriffen und teilweise zum Anlass genommen, eigene wohnungspolitische Anträge in den Stadtrat einzubringen.

Dies hat mich schließlich dazu veranlasst, einen eigenen Blog (stadtentwicklunggoettingen.wordpress.com) einzurichten, in dem ich meine Forschungsergebnisse breitenwirksam aufbereite und aktuelle wohnungspolitische Entwicklungen in der Stadt kommentiere. Flankiert habe ich diesen schließlich durch sehr knappe Einschätzungen zu aktuellen Medienberichten mit meinem Twitter-Account. Dies ermöglichte mir eine weitere Ausweitung meines Wirkungskreises, wie sich an dem breiten Feedback zeigte, dass ich von Parteien und lokalen Initiativen bekam. 
Abb. 2 Angebotsmietpreise in Göttingen 2019

Abb. 3 Entwicklung der Angebotsmietpreise in Göttingen zwischen 2013 und 2019

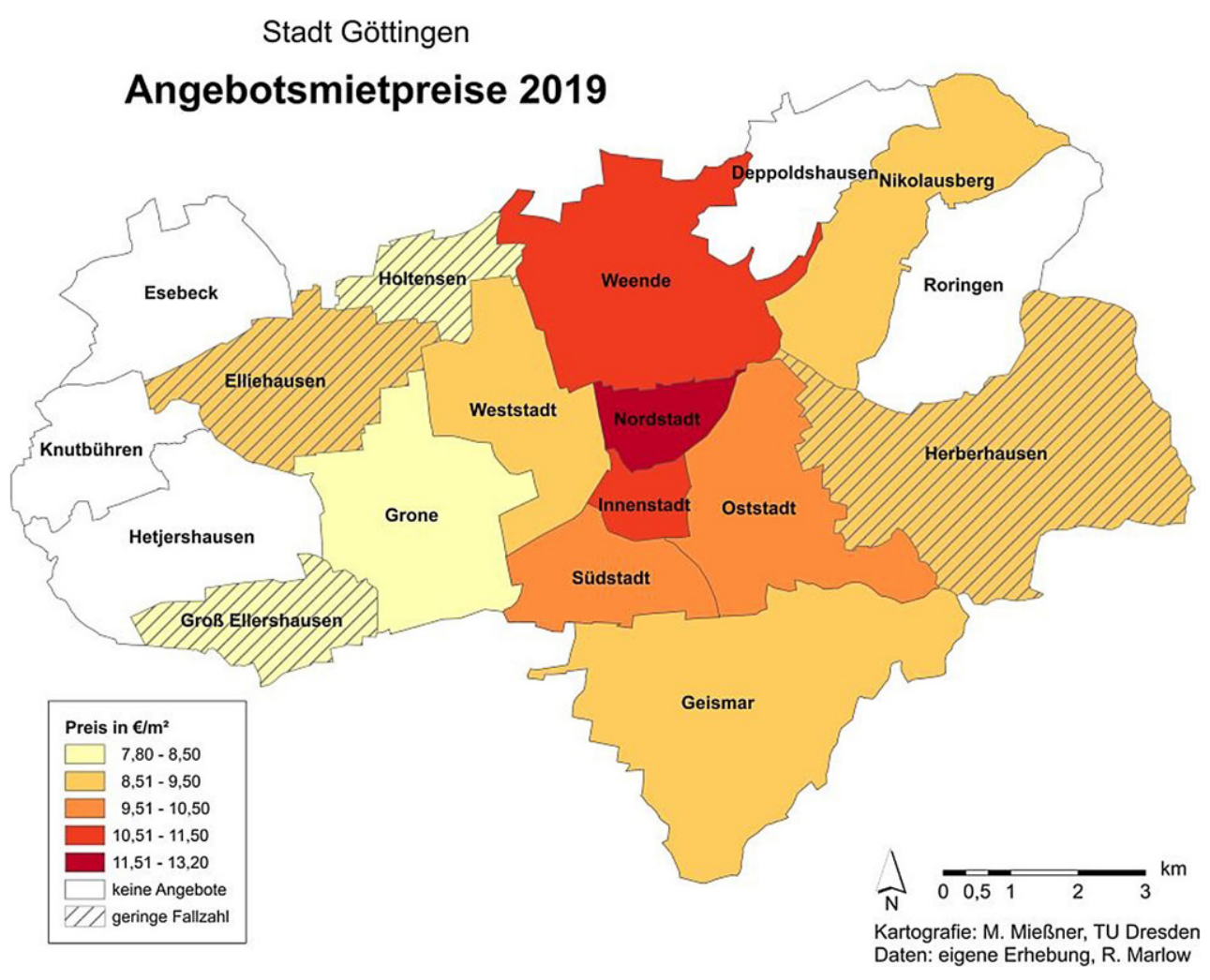

Stadt Göttingen

Entwicklung der Angebotsmietpreise

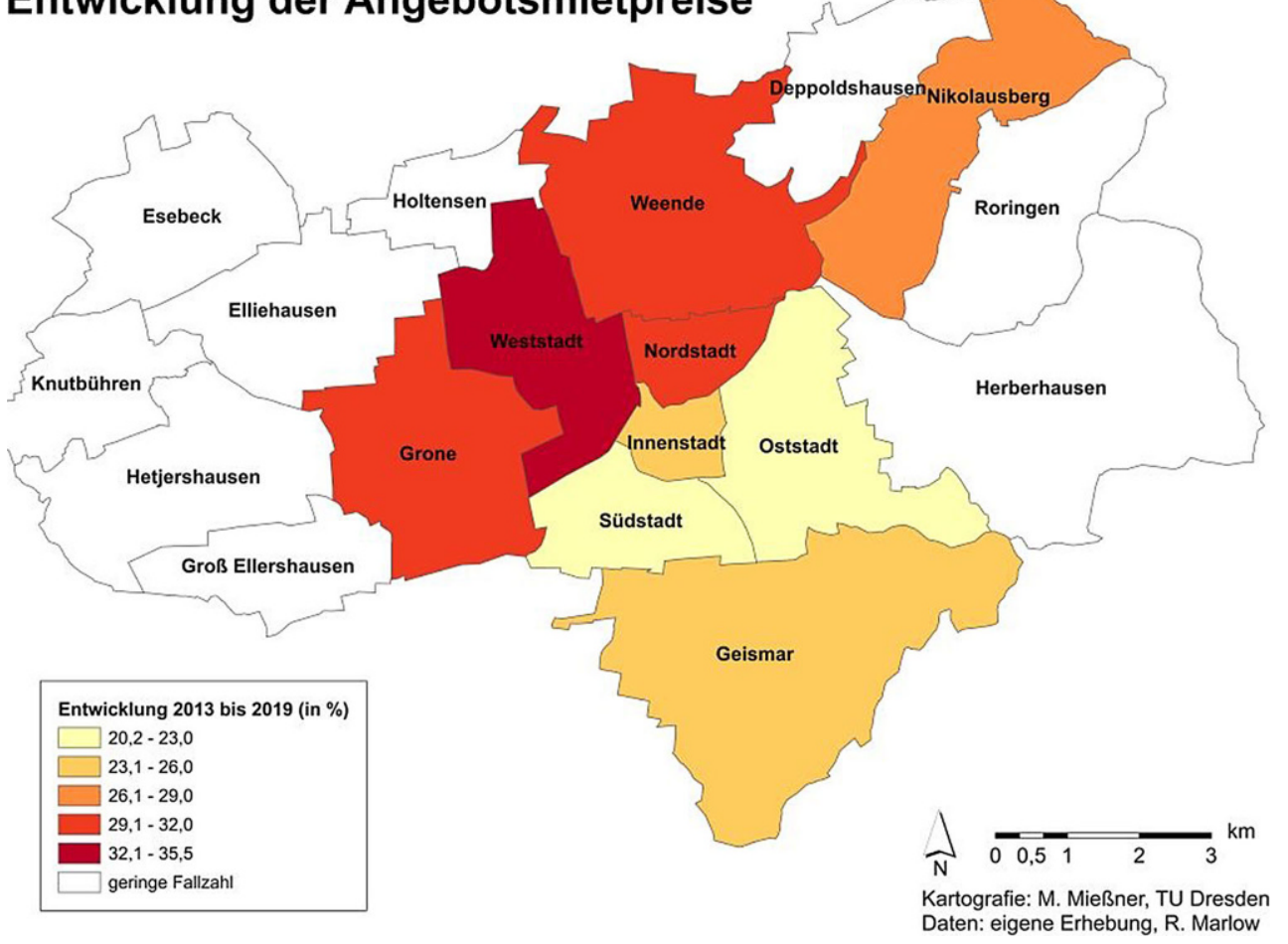


Abb. 4 Vonovia-Bestände in Göttingen in denen durch Modernisierung höhere Mietpreise erzielt werden sollen. (Foto: K. P. Wittemann)

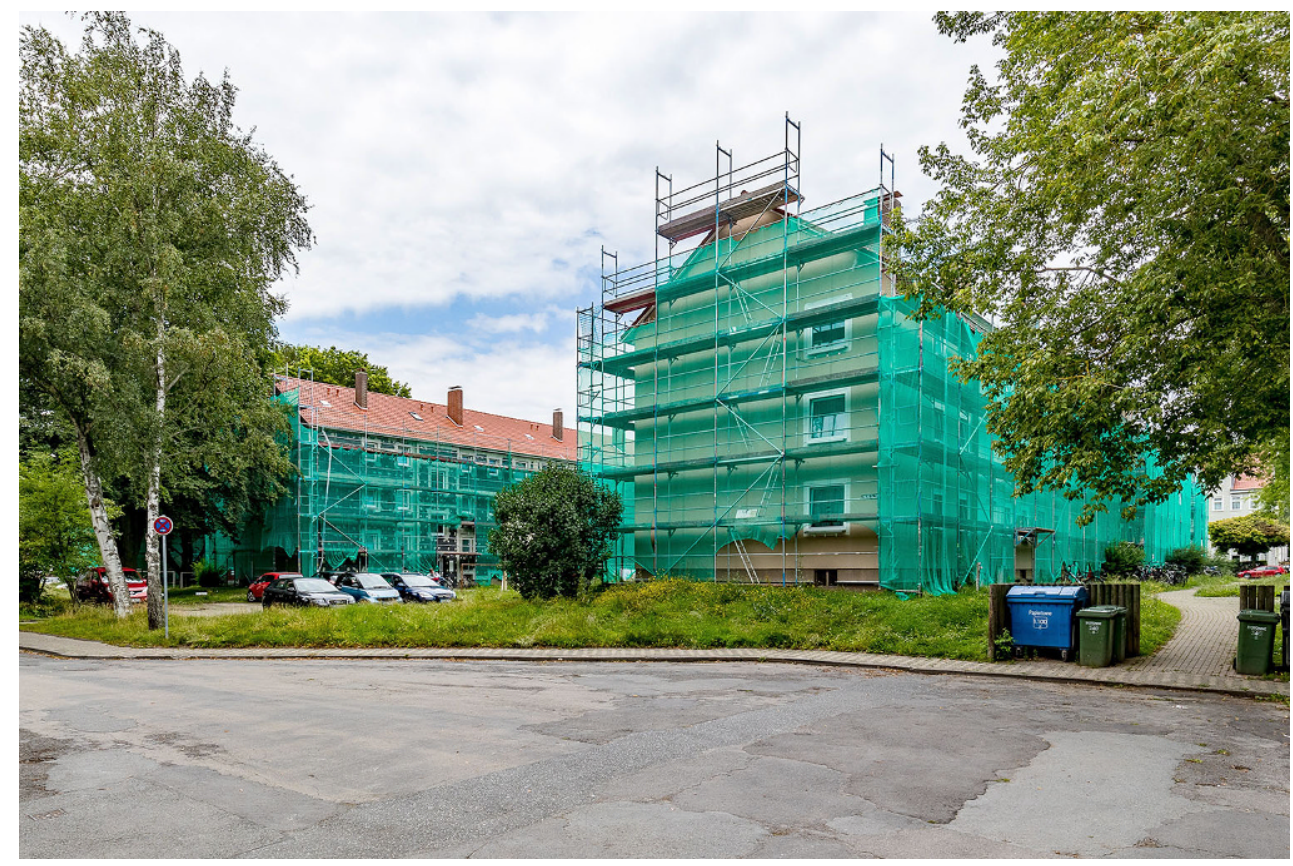

In diesen Transferformaten habe ich auch das oben erläuterte theoriegeleitete Verständnis der Göttinger Entwicklungen als finanzgetriebene Investition und als Studentifizierung aufbereitet. Im Sinne einer angewandten Kritischen Geographie konnte ich so einer interessierten Öffentlichkeit ermöglichen, ihre alltäglichen Erfahrungen einzuordnen und zu verstehen, wie es zur aktuellen Situation kommen konnte. Dies zeigte sich z. B. darin, dass einige zivilgesellschaftliche Akteure diese Prozesse bei ihren weiteren Initiativen berücksichtigten. So machte z. B. das Verständnis, dass Studierende nicht absichtlich und aktiv andere Bevölkerungsgruppen verdrängen, ein gemeinsames politisches Agieren unterschiedlicher Initiativen gegen diese Form der Wohnungsvergabe möglich. Dies dürfte eine Voraussetzung dafür gewesen sein, dass sich das Bündnis „Gutes Wohnen für Alle“ im Juni 2019 gründete, in dem verschiedene Akteure und Initiativen organisiert sind.

Ich nahm auch an mehreren Treffen lokaler wohnungspolitischer Initiativen teil. Ein enger Austausch hat sich mit dem Verein „In Grone, Verein für interkulturelle Nachbarschaft e. V." entwickelt. Der Verein ist in einem Wohngebiet aktiv, in dem im Jahr 2015 eine Tochterfirma der Adler Real Estate AG einen Bestand von über 1000 Wohneinheiten kaufte. Seitdem setzt die Adler AG in ihren Beständen u. a. mithilfe von Modernisierungsmaßnahmen erhebliche Mietpreissteigerungen durch. Der Verein befürchtet eine Verdrängung der bisherigen eher einkommensschwachen Bewohner*innenschaft. Mit dem Verein begann eine Zusammenarbeit, die im Transfer von Wissen über Strategien von Investoren, das Geschäftsgebaren finanzialisierter Unternehmen, der Beteiligung bei B-Planverfahren und in gemeinsamen Veranstaltungen bestand. Hilfreich für den Verein war dabei die Einschätzung, dass die Wachstumsstrategie von Adler seit 2016 in der Nachverdichtung bestand (Abb. 6). Darauf aufbauend adressierte der Verein nicht nur die Mieterhöhungen, sondern im B-Planverfahren auch die Nachverdichtung (Abb. 7). Nun waren die AdlerInteressen so in Gefahr, dass die Stadtverwaltung in den Konflikt eingriff und ihn befriedete (Mießner 2020a).

\section{Fazit}

Zusammenfassend kann konstatiert werden, dass es mir im Sinne der 3 zentralen Aspekte einer angewandten Kritischen Geographie, durchaus gelungen ist, die stärker theoriegeleiteten Forschungsergebnisse für eine breitere Öffentlichkeit zugänglich zu machen. Die bearbeiteten Forschungsfragen beschäftigten sich mit sozial relevanten Entwicklungen in der Stadt, weshalb auch der Wissenstransfer vielversprechend ausfiel. Besonders soziale Bewegungen, aber auch Parteien profitierten von den Ergebnissen und bezogen diese in ihre politischen Handlungen mit ein. Dennoch ist es wichtig, die eigene privilegierte Rolle als Wissenschaftler*in kritisch $\mathrm{zu}$ reflektieren, da diese einen recht prominenten Zugang zum öffentlichen Diskurs gewährt. Zu berücksichtigen ist außerdem, dass eine Spannung besteht zwischen den Bemühungen eine Nähe zu sozialen Bewegungen herzustellen und zugleich eine gewisse Distanz halten zu müssen. Es kann daher auch nicht Aufgabe von Wissenschaft sein für soziale Bewegungen zu sprechen. Eine kritische Wissenschaft kann letztere höchstens unterstützen. 


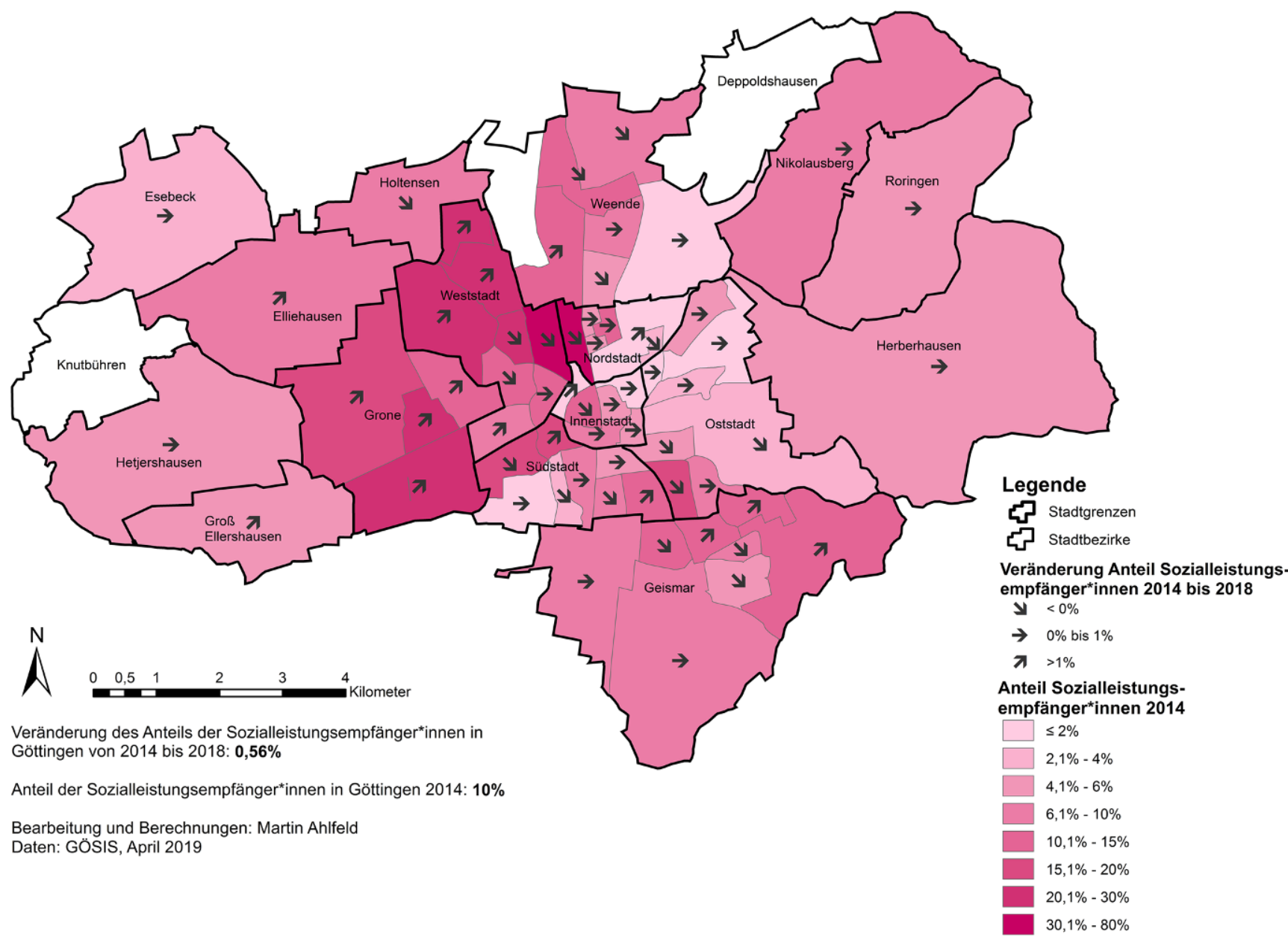

Abb. 5 Räumliche Verteilung und Entwicklung der Sozialleistungsempfänger*innen in Göttingen 2014 bis 2018

Abb. 6 Adler-Bestände, die durch Aufstockungen nachverdichtet werden sollen. (Foto: K.P. Wittemann)

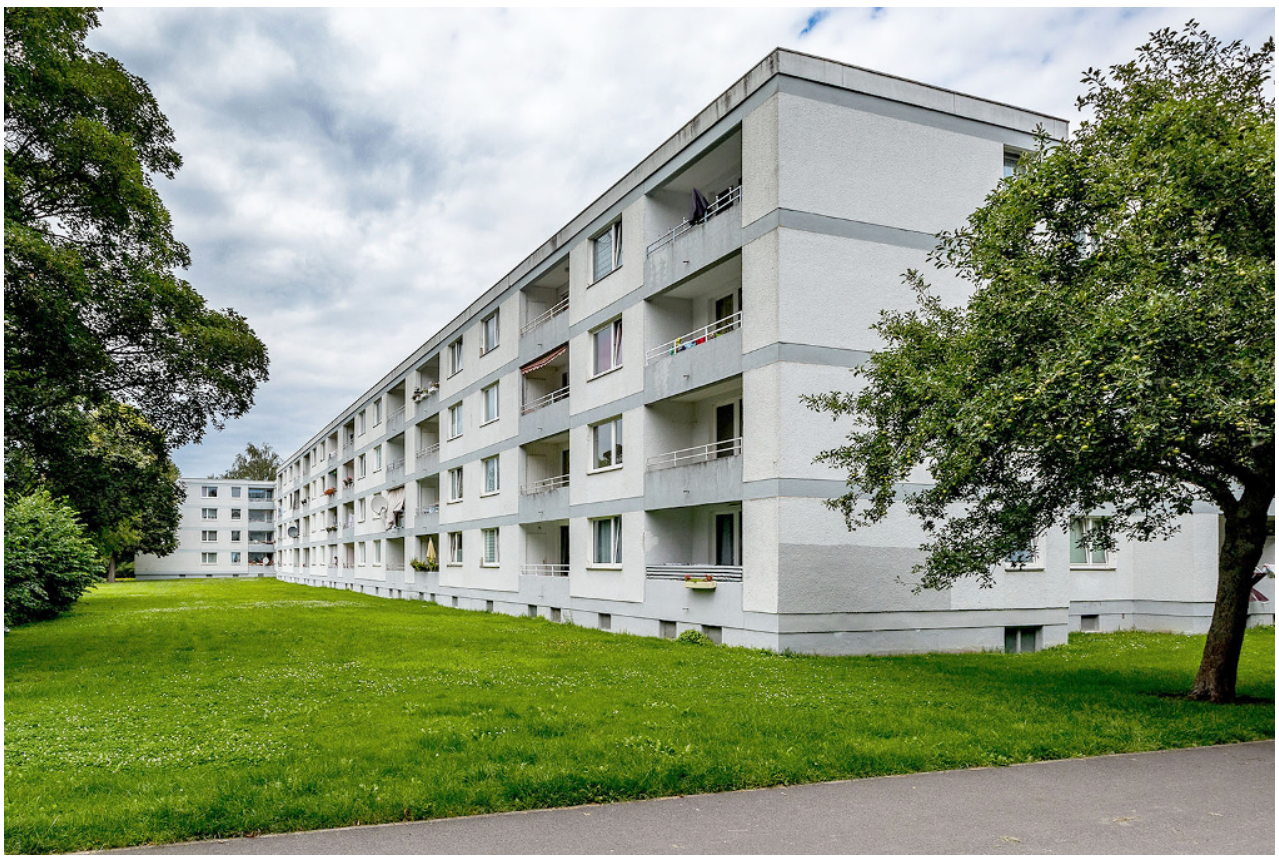


Abb. 7 Kundgebung gegen Mieterhöhungen in den AdlerBeständen vor dem Göttinger Rathaus am 08.11.2018. (Foto: K.P. Wittemann)

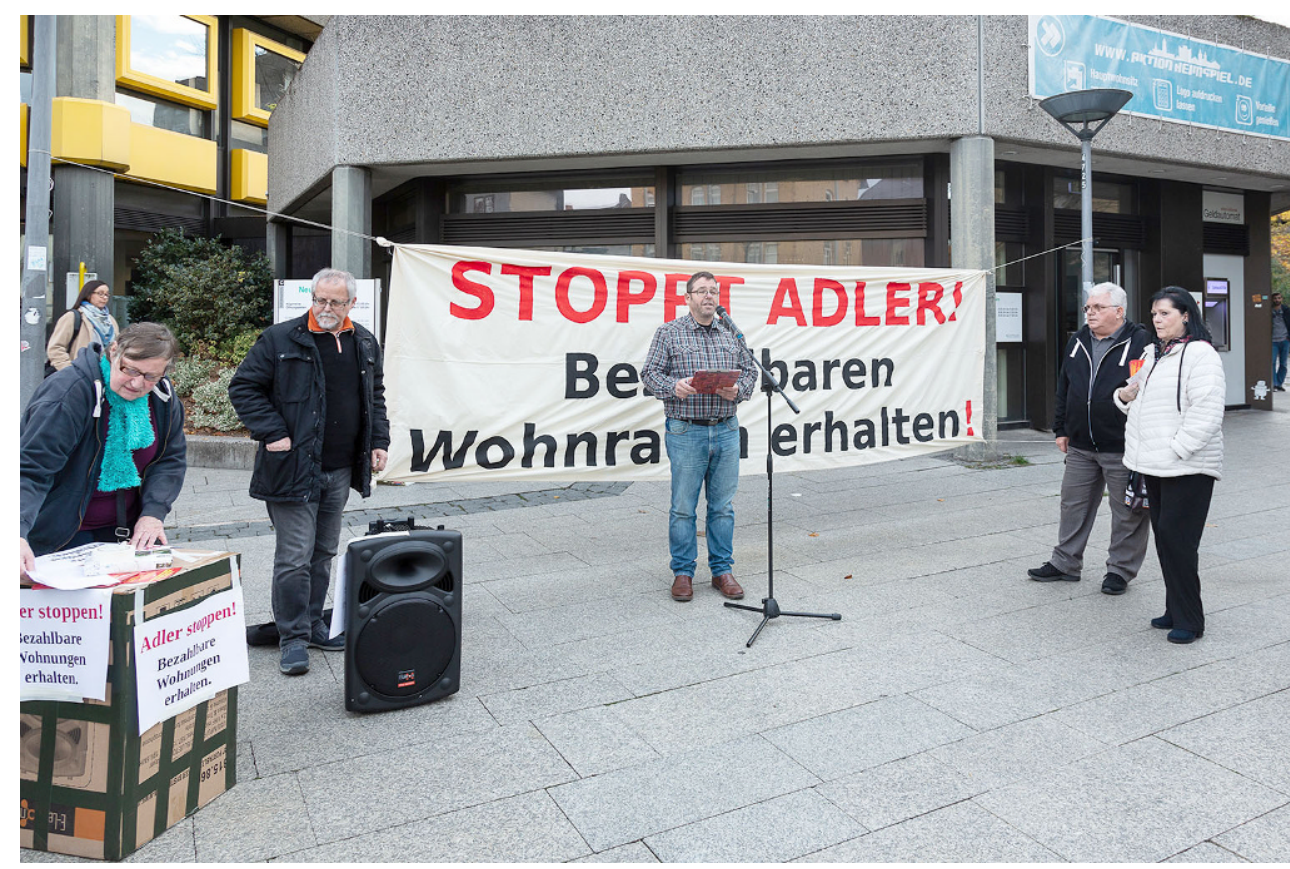

Es gibt aber auch Punkte, die eine so ausgerichtete angewandte Kritische Geographie erschweren. Zu nennen ist hier einerseits der hohe Arbeitsaufwand, der insbesondere durch den Kontakt mit den sozialen Bewegungen entsteht (vgl. Schipper 2017). Darüber hinaus sind lange nicht alle Ergebnisse, die eine praxisnahe Forschung generiert, geeignet, um in wissenschaftlichen Fachzeitschriften veröffentlicht zu werden. Nicht selten folgen die wichtigen theoretischen Debatten eines Faches ganz anderen Logiken (vgl. Carstensen et al. 2014) als die sozialen Fragen und Problemdiskurse vor Ort. Diese Diskrepanz auch institutionell (wieder) aufzuheben, ist daher eine zentrale Herausforderung einer angewandten Kritischen Geographie. In den bisherigen Arbeiten zur angewandten Kritischen Geographie scheint darüber hinaus, wenig konzeptionell gefasst zu sein, wie Verbesserungen der Lebensumstände von marginalisierten Bevölkerungsgruppen politisch befördert werden könnten. Natürlich steht die Forschung hier vor dem Problem, alternative Handlungsansätze in (lokale) Staatsapparate (Poulantzas 2002) einzuschreiben, während diesen zugleich eine strukturelle Selektivität zu eigen ist, die sie für progressive politische Kräfte wenig zugänglich macht. Diese Erfahrung musste auch ich in meiner eigenen Forschung machen, konnte aber immerhin erreichen, dass meine Forschungsergebnisse in Stadtrat und -verwaltung wahrgenommen und z. B. in die Begründung zum kommunalen Handlungsprogramm zur Sicherung und Schaffung bezahlbaren Wohnraums aufgenommen wurden. Zugleich wurden sie aber so kanalisiert, dass sie als Legitimation der neoliberalen städtischen Wohnungspolitik (Mießner 2020a) dienten. Zum Beispiel wurde in dem genannten kommunalen Hand- lungsprogramm nur auf meine Forschung zur Mietpreisentwicklung verwiesen, damit aber die Wohnungspolitik der allgemeinen Angebotsausweitung legitimiert. Meine verschiedenen Forschungserkenntnisse über die Notwendigkeit der Bereitstellung preisgünstigen Wohnraums oder stattfindende Segregations- und Verdrängungseffekte wurden jedoch nicht berücksichtigt. Der Herausforderung, auf politische Entscheidungen einzuwirken und zugleich der Gefahr der einseitigen Aufnahme eigener Forschungsergebnisse sollte sich aber eine angewandte Kritische Geographie dennoch stellen. Sie sollte versuchen, geeignete Umgangsstrategien mit diesem Problem zu entwickeln und darauf hinzuwirken auch politisch wirksam zu werden.

Funding Open Access funding provided by Projekt DEAL.

Open Access Dieser Artikel wird unter der Creative Commons Namensnennung 4.0 International Lizenz veröffentlicht, welche die Nutzung, Vervielfältigung, Bearbeitung, Verbreitung und Wiedergabe in jeglichem Medium und Format erlaubt, sofern Sie den/die ursprünglichen Autor(en) und die Quelle ordnungsgemäß nennen, einen Link zur Creative Commons Lizenz beifügen und angeben, ob Änderungen vorgenommen wurden.

Die in diesem Artikel enthaltenen Bilder und sonstiges Drittmaterial unterliegen ebenfalls der genannten Creative Commons Lizenz, sofern sich aus der Abbildungslegende nichts anderes ergibt. Sofern das betreffende Material nicht unter der genannten Creative Commons Lizenz steht und die betreffende Handlung nicht nach gesetzlichen Vorschriften erlaubt ist, ist für die oben aufgeführten Weiterverwendungen des Materials die Einwilligung des jeweiligen Rechteinhabers einzuholen.

Weitere Details zur Lizenz entnehmen Sie bitte der Lizenzinformation auf http://creativecommons.org/licenses/by/4.0/deed.de. 


\section{Literatur}

Aalbers M (2016) The financialization of housing; A political economy approach. Routledge, London, New York

Belina B, Naumann M, Strüver A (Hrsg) (2018) Handbuch Kritische Stadtgeographie. Westfälisches Dampfboot, Münster

Carstensen AL, Heimeshoff L-M, Jungehülsing J, Kirchhoff M, Trzeciak M (2014) Forschende Aktivist_innen und Aktivistische Forscher_innen: eine Hinleitung. In: Heimeshoff L-M, Hess S, Korn S, Schwenken H, Trzeciak M (Hrsg) Migration, Kontrolle, Wissen. Transnationale Perspektiven. Assoziation A, Berlin, S 257-268

Fehlberg T, Mießner M (2015) Mietpreissteigerungen und Wohnungsengpässe abseits der Ballungsräume; Investitionen in Wohnimmobilien in B-Lagen und Regionalzentren - das Beispiel Göttingen. sublurban 3:25-44

von Frieling H-D, Mießner M, Marlow R (2020) Wohnraumatlas Göttingen; Wohnungsversorgung und Wohnungspolitik in Göttingen seit 2010

GÖSIS (2019) Stadt Göttingen: Studierende und wohnberechtigte Bevölkerung 1950 bis 2018. https://duva-stg-extern.kdgoe.de/ Informationsportal/Dateien/055.01-WS-2018.pdf. Zugegriffen: 25. März 2020

Harvey D (2006) The limits to capital. Verso, London

Heeg S (2011) Finanzkrisen und städtische Immobilienmärkte. Die räumlichen Auswirkungen in und zwischen Städten. In: Demirović A, Dück J, Becker F, Bader P (Hrsg) VielfachKrise. Im finanzmarktdominierten Kapitalismus. VSA, Hamburg, S 181-198

Holm A, Schulz G (2016) GentriMap: Ein Messmodell für Gentrification und Verdrängung. In: Helbrecht I (Hrsg) Gentrifizierung in Berlin. Verdrängungsprozesse und Bleibestrategien. transcript, Bielefeld, S 287-318

Jonas AEG, McCann E, Thomas ME (2015) Urban geography; A critical introduction. Wiley-Blackwell, Hoboken

Kemper J, Wiegand F (2018) Marxistische Stadtforschung. In: Oßenbrügge J, Vogelpohl A (Hrsg) Theorien in der Raum- und Stadtforschung. Einführungen. Westfälisches Dampfboot, Münster, S 215-233

Lees L (2006) Gentrifying down the urban hierarchy; 'The cascade effect' in Portland, Maine. In: Bell D, Jayne M (Hrsg) Small cities. Urban experience beyond the metropolis. Routledge, London, S 91-104

Lefebvre H (2016) Das Recht auf Stadt. Edition Nautilus, Hamburg
Marlow R, Mießner M (2019) Mietpreisentwicklung 2019. https://stadtentwicklunggoettingen.wordpress.com/2019/12/ 04/mietpreisentwicklung-2019/. Zugegriffen: 25. März 2020

Mießner M (2018) Mietpreisentwicklungen 2018; Verknappung des Niedrigpreissegmentes nimmt weiter $\mathrm{zu}$. https://stadtentwicklunggoettingen.wordpress.com/2018/ 11/15/mietpreisentwicklungen-2018-verknappung-desniedrigpreissegmentes-nimmt-weiter-zu/. Zugegriffen: 25. März 2020

Mießner M (2019) Studentifizierung in Göttingen; Ein Beispiel für sozialräumliche Verdrängung in deutschen Universitätsstädten. Geogr Rundsch 10:22-27

Mießner M (2020a) Neoliberale Wohnungspolitik mit wohlfahrtsstaatlichen Versatzstücken; Das Beispiel der Universitätsstadt Göttingen. In: Rink D, Egner B (Hrsg) Lokale Wohnungspolitik. Beispiele aus deutschen Städten. Nomos, Baden Baden, S 269-286

Mießner M (2020b) Studentification in Germany: how investors generate profits from student tenants in Goettingen and the impacts on urban segregation. Eur Urban Reg Stud. https://doi.org/10.1177/ 0969776420934850

Mießner M, Klinge T (2017) Sozialräumliche Segregation in der Stadt Göttingen; Verdrängungsprozesse im Spannungsfeld von Investorenstrategien und sozialgerechter Wohnraumversorgung. In: Harteisen U, Dittrich C, Reeh T, Eigner-Thiel S (Hrsg) Tagungsband „Land und Stadt - Lebenswelten und planerische Praxis“, 24. November 2016. Tagungsband. Goltze, Göttingen, S 117-140

Oßenbrügge J, Vogelpohl A (Hrsg) (2018) Theorien in der Raum- und Stadtforschung; Einführungen. Westfälisches Dampfboot, Münster

Poulantzas N (2002) Staatstheorie; Politischer Überbau, Ideologie, autoritärer Etatismus. VSA, Hamburg

Sablowski T (2011) Krise und Kontinuität des finanzdominierten Akkumulationsregimes. Z Wirtschgeogr 55:50-64

Schipper S (2017) Wohnraum dem Markt entziehen?; Wohnungspolitik und städtische soziale Bewegungen in Frankfurt und Tel Aviv. Springer VS, Wiesbaden

Smith DP (2005) "Studentification": the gentrification factory? In: Atkinson R, Bridge G (Hrsg) Gentrification in a global context. The new urban colonialism. Routledge, London, S 72-86

Smith N (1979) Toward a theory of gentrification. A back to the city movement by capital, not people. J Am Plann Assoc 45:538-548 